\title{
Seguimento clínico laboratorial de trabalhadores que sofreram acidente com material biológico em instituições hospitalares
}

\author{
Clinical laboratory follow-up of workers who suffered accidents with biological material in hospital \\ institutions
}

Seguimiento de laboratorio clínico de trabajadores que sufrieron accidentes con material biológico en instituciones hospitalarias

\section{Mariana Guimarães Cardoso', Danielli Rafaeli Candido Pedro" , Raquel Gvozd Costa'II, Paloma de Souza Cavalcante Pissinati ${ }^{\mathrm{IV}}$, Mariana Ângela Rossaneis ${ }^{\mathrm{v}}$, Maria do Carmo Fernandez Lourenço Haddad ${ }^{\text {I }}$}

\begin{abstract}
Resumo: Objetivo: analisar o seguimento clínico laboratorial de trabalhadores que sofreram acidente com material biológico em instituições hospitalares (ATMB). Método: estudo transversal, quantitativo, com análise de prontuários de 550 trabalhadores que sofreram ATMB na área da $17^{\mathrm{a}}$ Regional de Saúde do Estado do Paraná, no período de outubro de 2010 a dezembro de 2013. Para a análise da associação foi utilizada a razão de prevalência (RP), e o teste de qui-quadrado de Wald para determinar se havia diferença entre as variáveis de estudo e o abandono do seguimento clínico. Resultados: dos acometidos, 51,5\% não deram seguimento clínico ao atendimento preconizado pelo protocolo de ATMB do Ministério da Saúde. Verificou-se maior abandono no tratamento quando não havia paciente. Conclusões: mais da metade dos acidentados não deram continuidade ao seguimento clínico, evidenciando a importância de estratégias para a saúde do trabalhador.
\end{abstract}

Descritores: Riscos ocupacionais; Saúde do trabalhador; Notificação de acidentes de trabalho; Enfermagem do trabalho

Abstract: Objective: To analyze the clinical laboratory follow-up of workers who suffered accidents with biological material in hospitals (ATMB). Method: cross-sectional, quantitative study with analysis of medical records of 550 workers who suffered ATMB in the area of the 17th Regional Health of the State of Paraná, from October 2010 to December 2013. For the analysis of the association was used the ratio of prevalence (PR), and

\footnotetext{
I Enfermeira. Mestre em Enfermagem pela Universidade Estadual de Londrina. São José do Rio Preto, São Paulo, Brasil. E-mail: mariguimaraes92@gmail.com. ORCID: https://orcid.org/0000-0002-6447-469X.

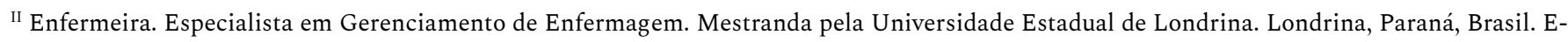
mail: danirafaeli@hotmail.com. ORCID: https://orcid.org/0000-0003-4141-1220.

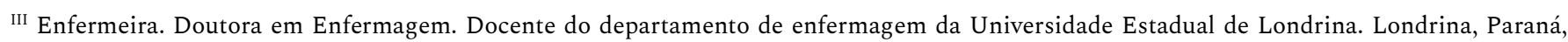
Brasil. E-mail: raquelgvozd@yahoo.com.br. ORCID: https://orcid.org/0000-0002-5816-8215.

IV Enfermeira. Doutora em Enfermagem. Diretora da Atenção Primária à Saúde da Secretaria Municipal de Saúde de Rolândia-PR. Londrina, Paraná, Brasil. E-mail: paloma_cavalcante_souza@hotmail.com. ORCID: https://orcid.org/0000-0001-9050-4330.

$\checkmark$ Enfermeira. Doutora em Enfermagem. Docente do departamento de enfermagem da Universidade Estadual de Londrina. Londrina, Paraná, Brasil. E-mail: marianarossaneis@gmail.com. ORCID: https://orcid.org/0000-0002-8607-0020.

${ }^{V I}$ Enfermeira. Doutora em Enfermagem. Docente Sênior do departamento de enfermagem da Universidade Estadual de Londrina. Londrina, Paraná, Brasil. E-mail: carmohaddad@gmail.com. ORCID: https://orcid.org/0000-0001-7564-8563.
} 
Wald's chi-square test to determine if there were differences between study variables and abandonment of clinical follow-up. Results: $51.5 \%$ of those affected did not give clinical follow-up to the care recommended by the Ministry of Health's ATMB protocol. There was greater abandonment of treatment when there was no patient. Conclusions: More than half of the injured did not continue the clinical follow-up, highlighting the importance of strategies for worker health.

Descriptors: Occupational Risks; Occupational Health; Occupational Accidents Registry; Occupational Health Nursing

Resumen: Objetivo: analizar el seguimiento en laboratorio clínico de trabajadores que sufrieron accidentes con material biológico en instituciones hospitalarias (ATMB). Método: estudio cuantitativo transversal con análisis de

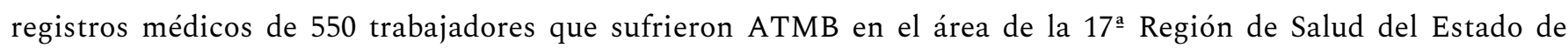
Paraná, desde octubre de 2010 hasta diciembre de 2013. Para el análisis de la asociación se utilizó la razón de prevalencia $(\mathrm{PR})$ y la prueba de chi-cuadrado de Wald para determinar si hubo diferencias entre las variables del estudio y el abandono del seguimiento clínico. Resultados: el 51.5\% de los afectados no dio seguimiento clínico a la atención recomendada por el protocolo ATMB del Ministerio de Salud. Hubo un mayor abandono del tratamiento cuando no hubo paciente. Conclusiones: más de la mitad de los lesionados no continuaron el seguimiento clínico, lo que destaca la importancia de las estrategias para la salud de los trabajadores.

Descripores: Riesgos Laborales; Salud Laboral; Notificación de Accidentes del Trabajo; Enfermería del Trabajo

\section{Introdução}

O Acidente de Trabalho com Material Biológico (ATMB) ocorre quando há contato percutâneo com sangue e/ou contato direto de mucosa ou pele não íntegra, com fluídos orgânicos potencialmente infectantes (sêmen, secreção vaginal, líquor, líquido sinovial, líquido pleural, peritoneal, pericárdico e amniótico) e fluídos orgânicos potencialmente não-infectantes (suor, lágrima, fezes, urina e saliva), exceto se contaminado com sangue. ${ }^{1}$

O registro do ATMB é exigido pela Lei nº 8.213/1991, por meio do Comunicado de Acidente de Trabalho (CAT), e deve ser realizado o preenchimento da ficha de notificação do Sistema de Informação de Agravos de Notificação (SINAN) para todos os trabalhadores, independentemente da existência de vínculo empregatício. ${ }^{2}$

Estima-se que, anualmente, ocorram aproximadamente, 3 milhões de exposições percutâneas entre os 35 milhões de profissionais da saúde de todo o mundo. ${ }^{3}$ Estes profissionais estão expostos a mais de 20 patógenos relacionados ao ATMB e entre eles, destacam-se o vírus da imunodeficiência humana (HIV), hepatite B (HBV) e hepatite C (HCV). ${ }^{1}$ 
3 | Cardoso MG, Pedro DRC, Costa RG, Pissinati PDC, Rossaneis MA, Haddad MCFL

O risco médio de transmissão do HIV é em torno de $0,3 \%$, do HCV varia entre 1 a $10 \%$ e do HBV de $6 \%$ a $30 \%$, podendo atingir até $62 \%$ quando o paciente-fonte tiver o antígeno do vírus da Hepatite B (HbsAg) positivo e nenhuma medida profilática for adotada após o acidente. Calculase que, anualmente, ATMB resultem em 15 mil infecções pelo $\mathrm{HCV}, 70$ mil pelo vírus da hepatite B (HBV) e 500 pelo HIV.3.-4

Existem diversas maneiras de se prevenir contra estes riscos biológicos, como o uso de equipamentos de proteção individual e a profilaxia pré-exposição da Hepatite $B$, por meio de vacina e/ou imunoglobulina, todavia, não existem vacinas para a Hepatite C. Caso o acidente com material biológico ocorra, existe ainda o uso da profilaxia pós-exposição (PPE) para a prevenção da contaminação pelo HIV que reduz a soroconversão em até $81,0 \%{ }^{5}$

Após a exposição decorrente de um ATMB, os profissionais devem procurar atendimento especializado a fim de detectar o seu status sorológico, o risco de infecção e receber o tratamento profilático, quando indicado, de acordo com o tipo de exposição. ${ }^{6}$

Entretanto, muitos profissionais não reconhecem sua vulnerabilidade à infecção e aos riscos ocupacionais advindos de um ATMB. ${ }^{4}$ Este tipo de acidente acarreta diversas consequências aos trabalhadores, tais como: preocupação, perda de sono, ansiedade, medo, descontrole emocional, culpa, problemas no relacionamento com a família e desconforto devido à quimioprofilaxia. ${ }^{7}$ Portanto, estudos identificaram que muitos profissionais acidentados não completam o seguimento clínico ou não realizam nenhuma medida profilática após exposição ocupacional., 8-9 Ressalta-se que o uso inadequado e/ou incompleto do esquema antirretroviral está diretamente relacionado ao risco de falha da profilaxia. ${ }^{10}$

Em uma revisão integrativa realizada sobre ATMB em profissionais da área da saúde, que reuniu 20 artigos científicos, demonstrou que, na maioria dos estudos, o acidente com material perfurocortante foi o tipo de acidente mais frequente e que a maioria dos profissionais realizam a notificação e iniciam o tratamento profilático ou curativo proposto, porém muitos não fazem o 
acompanhamento até o final e abandonam o tratamento. Além disso, esta revisão concluiu que os ATMB acontecem em quantidades relevantes e impactam negativamente a saúde do trabalhador, sendo prioritária a educação e conscientização dos profissionais de saúde quanto à prevenção dos acidentes e a adesão às condutas padronizadas após a exposição e ao tratamento completo quando indicado. ${ }^{11}$

Diante das complicações advindas do ATMB e da importância do seguimento adequado após exposição para a diminuição do risco de transmissibilidade de doenças, este estudo objetivou analisar o seguimento clínico laboratorial de trabalhadores que sofreram acidente com material biológico em instituições hospitalares.

\section{Método}

Estudo transversal de abordagem quantitativa. Foram analisados prontuários de 550 trabalhadores que sofreram ATMB em 21 municípios que compõe a $17^{\text {a }}$ Regional de Saúde do Estado do Paraná, no período de outubro de 2010 a dezembro de 2013, em um hospital estadual público que é referência para ocorrências desta natureza nesta regional.

Também foram coletadas informações em prontuários eletrônicos do Ambulatório do Consórcio Intermunicipal no qual os trabalhadores com potencial risco de contaminação, eram encaminhados pelo hospital para acompanhamento até a conclusão do caso. Além disso, nos casos dos prontuários que apresentaram dados incompletos, foram buscadas as informações nas fichas de notificação do Sistema de Informação de Agravos de Notificação (SINAN) armazenados no Núcleo de Atendimento ao Trabalhador da 17ª Regional de Saúde do Paraná.

Foram incluídos todos os trabalhadores que sofreram ATMB no período estabelecido e que tinham sido encaminhados para o serviço de referência para atendimento onde os prontuários eram captados. O critério de exclusão foram prontuários de estudantes da área da saúde, pois o 
5 | Cardoso MG, Pedro DRC, Costa RG, Pissinati PDC, Rossaneis MA, Haddad MCFL

acompanhamento desses casos não era no ambulatório onde os dados relacionados ao seguimento do tratamento foram coletados.

Os dados foram coletados entre os meses de dezembro de 2013 e junho de 2014. As variáveis de interesse do estudo foram: idade, sexo, nível de escolaridade, categoria profissional, mecanismos envolvidos no acidente (perfuração por agulha, corte, sangue sobre mucosa, apresentou pele íntegra ou pele lesada), ato e material do acidente e adesão ao seguimento clínico.

Considerou-se adesão ao seguimento clínico o comparecimento do profissional que sofreu exposição a material biológico a todos os agendamentos no ambulatório de referência, até a alta médica, conforme o protocolo estabelecido pelo Ministério da Saúde. ${ }^{12}$

O abandono do seguimento clínico foi considerado como a interrupção ou o não comparecimento aos atendimentos ambulatoriais.

Os dados coletados foram organizados em planilhas eletrônicas e analisados estatisticamente por meio do software Statistical Package for the Social Sciences (SPSS), versão 20.0. Foi realizada estatística descritiva para caracterizar os participantes quanto às variáveis selecionadas. Para a análise da associação, foi utilizada a razão de prevalência (RP). Realizou-se o teste de qui-quadrado de Wald, com nível de significância de 5\%, para determinar se havia diferença entre as variáveis de estudo e o abandono do seguimento clínico.

O estudo foi aprovado pelo Comitê de Ética em Pesquisa da Universidade Estadual de Londrina (UEL), CAAE n⿳0 19885813.1.0000.5231, respeitando as normas para o uso de dados em prontuários, conforme Resolução Conselho Nacional de Saúde nº 466/12.

\section{Resultados}

Dos 550 acometidos, $51,5 \%$ não deram seguimento clínico ao atendimento preconizado pelo protocolo de ATMB do Ministério da Saúde. ${ }^{10}$ 
Não houve associação entre as variáveis sociodemográficas e ocupacionais e o abandono do seguimento clínico. Porém, verificou-se que os trabalhadores do sexo masculino tiveram uma maior proporção de abandono do seguimento clínico $(53,4 \%)$ quando comparados ao sexo feminino (51,0\%). Com relação à escolaridade a maior proporção dos abandonos ocorreu em trabalhadores que atuavam em funções que exigiam apenas a escolaridade de nível técnico/auxiliar (51,9\%). A faixa etária em que houve maior percentual de abandono foi superior a 30 anos (50,5\%), com mediana de 34 anos. A maioria dos acidentes ocorreu entre profissionais de enfermagem $(73,9 \%)$, entretanto os trabalhadores de outras áreas profissionais tiveram maior percentual de abandono (56,7\%) (Tabela 1).

Tabela 1 - Associação entre variáveis sociodemográficas e ocupacionais de trabalhadores acometidos por acidentes com material biológico e o abandono do seguimento clínico. Londrina, PR, Brasil, 2010 - 2013.

\begin{tabular}{|c|c|c|c|c|c|c|}
\hline \multirow{2}{*}{$\begin{array}{l}\text { Dados } \\
\text { demográficos } \\
\text { ocupacionais }\end{array}$} & \multirow{2}{*}{$\begin{array}{r}\text { sócio } \\
\text { e }\end{array}$} & \multicolumn{2}{|c|}{$\begin{array}{c}\text { Abandono do seguimento } \\
\text { clínico }\end{array}$} & \multirow[t]{2}{*}{$\mathbf{R P}$} & \multirow[t]{2}{*}{$(\mathrm{IC}$ 95\%) } & \multirow[t]{2}{*}{ p-valor } \\
\hline & & $\begin{array}{c}\text { Sim } \\
\text { Frequência } \\
(\%)\end{array}$ & $\begin{array}{c}\text { Não } \\
\text { Frequência }(\%)\end{array}$ & & & \\
\hline \multicolumn{7}{|l|}{ Sexo } \\
\hline Feminino & & $228(51,0)$ & $219(49,0)$ & 1 & - & 0,662 \\
\hline Masculino & & $55(53,4)$ & $48(46,6)$ & 1,02 & $(0,92-1,11)$ & \\
\hline \multicolumn{7}{|c|}{ Escolaridade exigida para a função } \\
\hline Técnico/Auxiliar & & $216(51,9)$ & $200(48,1)$ & 1 & - & 0,644 \\
\hline Ensino Superior & & $66(46,6)$ & $67(50,4)$ & 1,02 & $(0,92-1,12)$ & \\
\hline \multicolumn{7}{|l|}{ Idade } \\
\hline Até 30 anos & & $108(50,5)$ & $106(49,5)$ & 1 & - & 0,712 \\
\hline 31 e mais & & $175(52,1)$ & $161(47,9)$ & 1,01 & $(0.93-1,10)$ & \\
\hline \multicolumn{7}{|c|}{ Área de atuação profissional } \\
\hline Outros & & $80(56,7)$ & $61(43,3)$ & 1,07 & $(0,97-1,18)$ & 0,146 \\
\hline Enfermagem & & $203(49,6)$ & $206(50,4)$ & 1 & - & \\
\hline
\end{tabular}

Fonte: Prontuários de trabalhadores que sofreram ATMB. 
7 | Cardoso MG, Pedro DRC, Costa RG, Pissinati PDC, Rossaneis MA, Haddad MCFL

A prevalência de abandono do seguimento clínico foi menor e estatisticamente significativa no conjunto de outros municípios, em relação ao município sede do centro de referência do atendimento $(\mathrm{RP}=0,85 / \mathrm{IC}=95 \%: 0,78-0,93)$. Também se verificou maior abandono no seguimento clínico quando não havia a identificação do paciente fonte, em comparação aqueles atendimentos em que a fonte era conhecida ( $\mathrm{RP}=1,20 / \mathrm{IC} 95 \%=1,08-1,34)$.

Tabela 2 - Associação entre os dados dos acidentes com material biológico e o abandono do seguimento clínico. Londrina, PR, Brasil, 2010 - 2013.

\begin{tabular}{|c|c|c|c|c|c|}
\hline \multirow[t]{2}{*}{ Dados do acidente } & \multicolumn{2}{|c|}{$\begin{array}{l}\text { Abandono do seguimento } \\
\text { clínico }\end{array}$} & \multirow[t]{2}{*}{$\mathbf{R P}$} & \multirow[t]{2}{*}{ (IC 95\%) } & \multirow[t]{2}{*}{ p valor } \\
\hline & $\begin{array}{c}\text { Sim } \\
\text { Frequência }(\%)\end{array}$ & $\begin{array}{c}\text { Não } \\
\text { Frequência }(\%)\end{array}$ & & & \\
\hline \multicolumn{6}{|l|}{ Exposição } \\
\hline Percutânea/Pele & $243(50,5)$ & $238(49,5)$ & 1 & - & 0,247 \\
\hline Mucosa & $40(58,0 \%)$ & $29(42,0)$ & 1,07 & $(0,95-1,22)$ & \\
\hline \multicolumn{6}{|l|}{ Material Orgânico } \\
\hline Sangue & $233(50,4)$ & $229(49,6)$ & 1 & - & 0,649 \\
\hline $\begin{array}{l}\text { Outros fluídos } \\
\text { corporais }\end{array}$ & $29(53,7)$ & $25(46,3)$ & 1,03 & $(0,87-1,19)$ & \\
\hline \multicolumn{6}{|c|}{ Circunstância do acidente } \\
\hline Adm. Medicamentos & $60(48,4)$ & $54(51,6)$ & 1 & - & 0,477 \\
\hline $\begin{array}{l}\text { Manipulação } \quad \text { de } \\
\text { Perfuro }\end{array}$ & $187(52,1)$ & $172(47,9)$ & $1,03(0,93-1,14)$ & & \\
\hline \multicolumn{6}{|l|}{ Agente agressor } \\
\hline Agulha & $215(50,7)$ & $209(49,3)$ & 1 & - & 0,520 \\
\hline Outros & $68(54,0)$ & $58(46,0)$ & 1,03 & $(0,93-1,14)$ & \\
\hline \multicolumn{6}{|l|}{ Fonte conhecida } \\
\hline $\operatorname{Sim}$ & $220(48,2)$ & $236(51,8)$ & 1 & - & $<0,001$ \\
\hline Não & $63(67,0)$ & $31(33,0 \%)$ & 1,20 & $(1,08-1,34)$ & \\
\hline \multicolumn{6}{|l|}{ Município do acidente } \\
\hline Londrina & $208(56,7)$ & $159(43,3)$ & 1 & - & $<0,001$ \\
\hline
\end{tabular}




\begin{tabular}{lllll}
\hline Outros & $75(41,0)$ & $108(59,0)$ & 0,85 & $(0,78-0,93)$ \\
\hline
\end{tabular}

Fonte: Prontuários de trabalhadores que sofreram ATMB.

No que se refere a análise do seguimento clínico, identificou-se que a maioria dos trabalhadores abandonaram o seguimento clínico após a primeira consulta ambulatorial e menor prevalência de abandono nos casos em que era necessário o uso de antirretroviral $(\mathrm{RP}=0,83 / \mathrm{IC} 95 \%=0,75-0,92)$ (Tabela 3).

Tabela 3 - Associação entre os dados do seguimento ambulatorial dos acidentados com material biológico e o abandono do seguimento clínico. Londrina, PR, Brasil, 2010 - 2013.

\begin{tabular}{|c|c|c|c|c|c|c|}
\hline \multirow[t]{2}{*}{$\begin{array}{l}\text { Dados do } \\
\text { clínico }\end{array}$} & \multirow[t]{2}{*}{ tratamento } & \multicolumn{2}{|c|}{$\begin{array}{c}\text { Abandono do seguimento } \\
\text { clínico }\end{array}$} & $\mathbf{R P}$ & (IC 95\%) & p valor \\
\hline & & $\begin{array}{l}\text { Sim } \\
\text { Frequência } \\
(\%)\end{array}$ & $\begin{array}{c}\text { Não } \\
\text { Frequência }(\%)\end{array}$ & & & \\
\hline
\end{tabular}

\begin{tabular}{|c|c|c|c|c|c|}
\hline & ulatoriais & & & \multirow[b]{3}{*}{$(0,81-1,0)$} & \multirow[b]{3}{*}{0,069} \\
\hline Uma consulta & $89(53,0)$ & $79(47,0)$ & - & & \\
\hline \multirow[t]{2}{*}{ Duas ou mais } & $71(43,0)$ & $94(57,0)$ & 0,90 & & \\
\hline & roviral & & & & \\
\hline Sim & $37(34,3)$ & $71(65,7)$ & - & & \multirow[b]{2}{*}{0,001} \\
\hline Não & $230(52,0)$ & $212(48,0)$ & 0,83 & $(0,75-0,92)$ & \\
\hline
\end{tabular}

Fonte: Prontuários de trabalhadores que sofreram ATMB.

\section{Discussão}

Os resultados desse estudo demonstraram que mais da metade dos profissionais acidentados com material biológico abandonaram o seguimento clínico.

O acometimento de 550 trabalhadores no período de três anos é alarmante, indicando a necessidade, por parte dos gestores, do desenvolvimento e implantação de práticas institucionais de prevenção de acidentes, constante avaliação dos riscos a que seus funcionários estão expostos, investindo na minimização dos mesmos e promovendo constantes ações educativas em saúde. ${ }^{9}$ 
9 | Cardoso MG, Pedro DRC, Costa RG, Pissinati PDC, Rossaneis MA, Haddad MCFL

Quanto aos fatores sociodemográficos dos trabalhadores que sofreram ATMB, observou-se que houve predominância do sexo feminino, adultos jovens (30-39 anos) e trabalhadores da equipe de enfermagem. O maior acometimento de ATMB em mulheres coaduna ao encontrado em outros estudos que avaliaram que as mulheres predominam entre os profissionais de saúde, em especial na área de enfermagem. ${ }^{13-15}$ Entretanto, o sexo masculino demonstrou maior percentual de abandono do seguimento clínico e este fato pode ser justificado por barreiras socioculturais que estão relacionadas ao homem como ser forte, viril e invulnerável, bem como as questões culturais de padrões de masculinidade que reprimem as necessidades e os cuidados com a saúde. ${ }^{16}$

Tais dados demonstram a necessidade de recorrentes capacitações e treinamentos relacionados a prevenção de ATMB e também a importância da continuidade do acompanhamento e dos riscos do abandono do tratamento para a saúde do trabalhador, sendo papel do gestor, a coordenação de uma equipe que desenvolva suas atividades respeitando os preceitos éticos e técnicos da profissão, trabalhando diariamente para a minimização de riscos e danos. ${ }^{13-14}$

Os trabalhadores de nível técnico/auxiliares da equipe de enfermagem foram os mais acometidos por acidentes com material biológico, como o encontrado em outros estudos que apresentam a categoria técnico/auxiliar de enfermagem como a maior categoria da área da saúde a ter contato direto com o paciente. ${ }^{17-18}$ Todavia, os profissionais da equipe de enfermagem demonstraram menor percentual de abandono $(49,6 \%)$ que os outros profissionais da área da saúde $(56,7 \%)$, o que pode ser atribuído ao profissional de enfermagem ter maior aproximação com os possíveis desfechos do ATMB para sua saúde e serem melhor preparados para tal situação em seu curso. $^{7}$

Por outro lado, observou-se que os profissionais de nível técnico de enfermagem tiveram um maior percentual de abandono quando comparados aos enfermeiros, dado possivelmente 
atribuído aos cursos de graduação prepararem seus alunos para lidar com este acidente de trabalho. Estudo que avaliou os fatores que interferem no atendimento do ATMB, identificou o conhecimento técnico científico dos profissionais da saúde como facilitador para o sucesso de atendimento pós-exposição. ${ }^{19}$

Com relação à idade, os profissionais com idade acima de 31 anos tiveram maior percentual de abandono do seguimento clínico e infere-se que profissionais com idade superior e há mais tempo na profissão, podem ter passado por mais de um acidente de trabalho e com isso descredenciam o tratamento. Ressalta-se que profissionais mais experientes podem ser mais resistentes a mudanças em seu comportamento. ${ }^{4}$

No que refere às circunstâncias do acidente, o município onde o estudo foi realizado apresentou associação significativa com o maior abandono do seguimento clínico, sendo que 94,5\% dos trabalhadores sofreram acidentes percutâneos, que são considerados exposições biológicas graves. Infere-se que os profissionais advindos de outros locais demonstraram maior comprometimento e preocupação com o acidente, dando continuidade ao seguimento clínico.

Ressalta-se que acidentes ocorridos nos municípios vizinhos podem apresentar um maior índice de subnotificação, já que estes profissionais possuem menor acessibilidade ao hospital de referência da $17^{a}$ Regional e ao Ambulatório, além da demora resultante da distância percorrida para iniciar o atendimento que, muitas vezes, supera as duas horas recomendadas para o início da quimioprofilaxia.

O protocolo do Ministério da Saúde garante atendimento aos ATMB em qualquer pronto socorro, ${ }^{13}$ entretanto, nem todos os estabelecimentos de saúde possuem médicos capacitados e materiais, como medicamentos de quimioprofilaxia, sendo necessário o encaminhamento dos trabalhadores acidentados.

A maioria ATMB decorreu do manuseio de perfurocortantes, especialmente, com agulhas. Foram considerados, nesta categoria, procedimentos que envolviam punção venosa/arterial, 
11 | Cardoso MG, Pedro DRC, Costa RG, Pissinati PDC, Rossaneis MA, Haddad MCFL

descarte, reencape e manipulação de caixa com materiais perfurocortantes. Estes achados são semelhantes a resultados de um estudo no qual foi identificado que a maioria dos acidentes ocorreu por contato com agulha durante ou após o seu uso ou na tentativa de reencape destes materiais. ${ }^{20}$

Além disso, no presente estudo identificou-se que o abandono do seguimento esteve associado ao uso de quimioprofilaxia. De acordo com o Ministério da Saúde, aproximadamente, um terço dos profissionais interrompem o tratamento devido aos efeitos adversos à quimioprofilaxia antirretroviral. ${ }^{10}$

Destaca-se o fato de que a quimioprofilaxia para HIV, quando indicada, tem uma relação de tempo-dependência, sendo o seu maior benefício quando iniciada até duas horas após o acidente. ${ }^{12}$ Embora sejam discretos os efeitos gastrointestinais, cefaleia e fadiga; bem como as alterações laboratoriais, observa-se que quanto maior a interferência dos antirretrovirais nas atividades cotidianas e mais incômodos os efeitos adversos, maior será o risco de não adesão à profilaxia. $^{10}$ Estudo demonstrou a importância da quimioprofilaxia pelo uso de zidovudina (AZT), que pode reduzir em até $81 \%$ o risco de soroconversão após a exposição ocupacional ao material biológico. ${ }^{13}$

Com relação ao risco de exposição dos trabalhadores acidentados a soroconversão, estudo realizado nos Estados Unidos entre 1981 a 2010 demonstrou que 57 trabalhadores norteamericanos sofreram soroconversão, após acidente do trabalho com exposição a material biológico potencialmente contaminado. ${ }^{21}$

Embora as políticas públicas de saúde brasileiras invistam na temática por meio de Centros de Referência em Saúde do Trabalhador - CEREST, estabelecimento de notificação compulsória pelo SINAN pós agravos de ATMB e a implantação de protocolos, como a NR-32, que estimulam medidas de precauções-padrão com o objetivo de proteger profissionais de saúde contra possível contaminação ocupacional, não há dados precisos sobre o número de 
ocorrências de soroconversão ao vírus HIV, Hepatite B e C entre os profissionais de saúde brasileiros. $^{22}$

Para a coleta de dados encontrou-se algumas limitações, sendo a principal delas a falta de informações detalhadas nos prontuários que permitissem identificar a caracterização do ATMB, bem como algumas informações que indicassem os motivos da descontinuidade do tratamento. Essas limitações demonstram o quanto o atendimento ao trabalhador que sofre um ATMB ainda não atende a legislação trabalhista, principalmente em municípios de médio e pequeno porte.

\section{Considerações finais}

O elevado índice de não adesão ao seguimento clínico, em especial nos profissionais de saúde que tiveram a indicação de quimioprofilaxia é preocupante, considerando que a utilização de antirretrovirais poderia reduzir, de maneira significativa, a soroconversão de infecções advindas de contaminação ocupacional.

Estes achados reafirmam a necessidade de maior atenção ao seguimento clínico dos profissionais de saúde após exposição à ATMB. Assim, sugere-se a implantação de medidas que facilitem o acesso ao acompanhamento dos casos no local de origem do trabalhador.

Ressalta-se que para garantir adesão ao tratamento, as instituições de saúde devem estar capacitadas para acolher o profissional acometido de maneira efetiva, sanando-lhe as dúvidas quanto aos efeitos colaterais da quimioprofilaxia, exames sorológicos que devem ser realizados e periodicidade de retornos ambulatoriais. Todas estas estratégias devem ser atreladas, no intuito de acompanhar o trabalhador durante todo o seguimento clínico, dando-lhe o suporte necessário.

Sugere-se o desenvolvimento de outras pesquisas nesta temática que possam ofertar um panorama de como os ATMB vem acontecendo, a ocorrência de abandono do tratamento e de que forma está acontecendo o acompanhamento desses profissionais. Acredita-se que, com base 
em futuros estudos, será possível a construção de medidas cada vez mais sólidas em busca da redução de acidentes e da melhoria da qualidade de vida do trabalhador.

\section{Referências}

1. Marziale MHP, Santos HEC, Trovó MEM. Consequências individuais e ocupacionais da exposição a material biológico entre trabalhadores de enfermagem. Rev Enferm UERJ [Internet]. 2015 jul-ago [acesso em 2018 nov 28];23(4):449-54. Disponível em: https://www.epublicacoes.uerj.br/index.php/enfermagemuerj/article/view/9481 doi: 10.12957/reuerj.2015.9481

2. Oliveira EC, Ponte MAC, Dias MSA, Silva ASR, Torres ARA, Ferreira VES. Análise epidemiológica de acidentes de trabalho com exposição a material biológico entre profissionais de enfermagem. SANARE (Sobral) [Internet]. 2015 jan-jun [acesso em 2018 nov 28];14(1):27-32. Disponível em: https://sanare.emnuvens.com.br/sanare/article/download/603/320

3. Araújo TME, Silva NC. Acidentes perfurocortantes e medidas preventivas para hepatite B adotadas por profissionais de enfermagem nos serviços de urgência e emergência de Teresina, Piauí. Rev Bras Saúde Ocup [Internet]. 2014 jul-dez [acesso em 2018 nov 28];39(130):175-83. Disponível em: http://www.scielo.br/scielo.php?pid=S0303-76572014000200175\&script=sci_abstract\&tlng=es doi: 10.1590/0303-7657000079413.

4. Teresa V, Elena V, Alberto F, Jeannette D. Exposure to risk fluids in health personnel. Evaluation of direct costs in their management. Rev Chilena Infectol [Internet]. 2018 [acesso em 2019 abr 16];35(5):4907. Disponível em: https://www.ncbi.nlm.nih.gov/pubmed/30724995 doi: 10.4067/s0716-10182018000500490

5. Julio RS, Filardi MBS, Marziale MHP. Acidentes de trabalho com material biológico ocorridos em municípios de Minas Gerais. Rev Bras Enferm [Internet]. 2014 jan-fev [acesso em 2018 nov 28];67(1):11926. Disponível em: http://www.scielo.br/scielo.php?pid=S003471672014000100119\&script=sci_abstract\&tlng=pt doi: 10.5935/0034-7167.20140016

6. Luize PB, Canini SRMS, Gir E, Toffano SEM. Condutas após exposição ocupacional a material biológico em um hospital especializado em oncologia. Texto \& Contexto Enferm [Internet]. 2015 jan-mar [acesso em 2018 nov 28];24(1):170-7. Disponível em: http://www.scielo.br/pdf/tce/v24n1/pt_0104-0707-tce24-01-00170.pdf doi: 10.1590/0104-07072015002700013

7. Marziale MHP, Santos HEC, Cenzi CM, Rocha FLR, Trovó MEM. Consequências da exposição ocupacional a material biológico entre trabalhadores de um hospital universitário. Esc Anna Nery Rev Enferm [Internet]. 2014 jan-mar [acesso em 2018 nov 28];18(1):11-6. Disponível em: http://www.scielo.br/scielo.php?pid=S1414-81452014000100011\&script=sci_abstract\&tlng=pt doi:10.5935/1414-8145.20140002 
8. Porto JS, Marziale MHP. Motivos e consequências da baixa adesão às precauções padrão pela equipe de enfermagem. Rev Gaúch Enferm [Internet]. 2016 jun [acesso em 2018 nov 28];137(2):1-15. Disponível em: http://www.scielo.br/scielo.php?script=sci_arttext\&pid=S1983-14472016000200501 doi: 10.1590/19831447.2016.02.57395

9. Carvalho DC, Rocha JC, Gimenes MCA, Santos EC, Valim MD. Acidentes de trabalho com material biológico na equipe de enfermagem de um hospital do Centro-Oeste brasileiro. Esc Anna Nery Rev Enferm [Internet]. 2018 [acesso em 2018 nov 28];22(1):20170140. Disponível em: http://www.scielo.br/scielo.php?script=sci_arttext\&pid=S1414-81452018000100206 doi: 10.1590/21779465-EAN-2017-0140

10. Ministério da Saúde (BR), Secretaria de Vigilância em Saúde. Recomendações para atendimento e acompanhamento de exposição ocupacional a material biológico: HIV e hepatites B e C [Internet]. Brasília (DF); 2017 [acesso em 2018 nov 28]. Disponível em: http://bvsms.saude.gov.br/bvs/publicacoes/04manual_acidentes.pdf

11. Carvalho TS, Luz RA. Acidentes biológicos com profissionais da área da saúde no Brasil: uma revisão da literatura. Arq Méd Hosp Fac Ciênc Méd Santa Casa São Paulo [Internet]. 2018 [acesso em 2018 abr 16];63(1):31-6. Disponível em: http://arquivosmedicos.fcmsantacasasp.edu.br/index.php/AMSCSP/article/view/61 doi: https://doi.org/10.26432/1809-3019.2018.63.1.31

12. Ministério da Saúde (BR), Comissão Nacional de Incorporação de Tecnologias no SUS (CONITEC). Protocolo clínico e diretrizes terapêuticas para profilaxia pós exposição de risco à infecção pelo HIV, IST e hepatites virais [Internet]. Brasília (DF); 2017 [acesso em 2018 nov 28]. Disponível em: http://www.aids.gov.br/pt-br/pub/2015/protocolo-clinico-e-diretrizes-terapeuticas-para-profilaxia-posexposicao-pep-de-risco

13. Teles AFS, Ferreira MPS, Coelho TCB, Araújo TM. Acidentes de trabalho com equipe de enfermagem: uma revisão crítica. Rev Saúde Colet UEFS [Internet]. 2016 jun [acesso em 2018 nov 28];6(1):62-8. Disponível em: http://periodicos.uefs.br/index.php/saudecoletiva/article/view/1082 doi: 10.13102/rscdauefs.v6i1.1082

14. Lopes JSP, Carvalho TES, Nascimento JF, Alves CAS, Pereira AKP, Rodrigues TS. Características dos acidentes de trabalho com material biológico em profissionais de enfermagem. Rev Eletrônica Acervo Saúde [Internet]. 2017 [acesso em 2018 nov 2018];9(3):1178-86. Disponível em: https://www.acervosaude.com.br/doc/34_2017.pdf

15. La Torre G, Scalingi S, Garruto V, Siclari M, Chiarini M, Mannocci A. Knowledge, attitude and behaviours towards recommended vaccinations among healthcare workers. Healthcare (Basel) [Internet]. 2017 mar [acesso em 2019 abr 16];5(1):13. Disponível em: https://www.mdpi.com/2227-9032/5/1/13 doi: 10.3390/healthcare5010013 
16. Siqueira, BPJ, Teixeira JRB, Valença Neto PF, Boery EN, Vilela ABA. Homens e cuidado à saúde nas representações sociais de profissionais de saúde. Esc Anna Nery Rev Enferm [Internet]. 2014 out-dez [acesso em 2018 nov 28];18(4):690-6. Disponível em: http://www.scielo.br/pdf/ean/v18n4/1414-8145-ean18-04-0690.pdf doi:10.5935/1414-8145.20140098

17. Gonçalves PM, Belfort IK, Fernandes MA, Monteiro SCM, Sousa WR, Sampaio RM. Análise da estatística de acidentes com exposição de material biológico no Maranhão nos anos 2009-2010. Rev Pesqui Saúde [Internet]. 2014 set-dez [acesso em 2018 nov 28];15(3):360-3. Disponível em: http://www.periodicoseletronicos.ufma.br/index.php/revistahuufma/article/view/3660/1667

18. Santos Junior EP, Batista RRAM, Almeida ATF, Abreu RAA. Acidente de trabalho com material perfurocortante envolvendo profissionais e estudantes da área da saúde em hospital de referência. Rev Bras Med Trab [Internet]. 2015 [acesso em 2018 nov 28];13(2):69-75. Disponível em: http://www.rbmt.org.br/details/6/pt-BR/acidente-de-trabalho-com-material-perfurocortante-envolvendoprofissionais-e-estudantes-da-area-da-saude-em-hospital-de-referencia

19. Ribeiro LCM, Souza ACS, Tipple AFV, Melo DS, Peixoto MKAV, Munari DB. Fatores intervenientes no fluxo de atendimento ao profissional acidentado com material biológico. Rev Esc Enferm USP [Internet]. 2014 [acesso em 2018 nov 28];48(3):507-13. Disponível em: http://www.scielo.br/scielo.php?pid=S0080-62342014000300507\&script=sci_arttext\&tlng=pt doi: 10.1590/S0080-623420140000300017

20. Sarsangi V, Salehiniya H, Hannani M, Marzaleh MA, Abadi YS, Honarjoo F, et al. Assessment of workload effect on nursing occupational accidents in hospitals of Kashan, Iran. Biomed Res Ther [Internet]. 2017 [acesso em 2018 nov 28];4(8):1527-40. Disponível em: http://www.bmrat.org/index.php/BMRAT/article/view/226 doi: 10.15419/bmrat.v4i08.226

21. Centers for Disease Control and Prevention (CDC), Department of Health and Human Services (US). Recommendations for HIV prevention with adults and adolescents with HIV in the United States, 2014 [Internet]. Atlanta: Centers for Disease Control and Prevention; 2014 [acesso em 2018 nov 28]. Disponível em: https://stacks.cdc.gov/view/cdc/44064

22. Valim MD, Marziale MHP, Hayashida M, Richart-Martínez M. Ocorrência de acidentes de trabalho com material biológico potencialmente contaminado em enfermeiros. Acta Paul Enferm [Internet]. 2014 maio-jun [acesso em 2018 nov 28];27(3):280-6. Disponível em: http://www.scielo.br/scielo.php?pid=S010321002014000300280\&script=sci_abstract\&tlng=pt doi: 10.1590/1982-0194201400047

\section{Autor correspondente}

Nome: Mariana Guimarães Cardoso

E-mail: mariguimaraes92@gmail.com

Endereço: Rua Alexandre Marini, 175 - Dom Lafayte Libanio. São José do Rio Preto-SP.

CEP: 15046050 


\section{Contribuições de Autoria}

\section{1 - Mariana Guimarães Cardoso}

Contribuições: concepção e planejamento do projeto de pesquisa, obtenção ou análise e interpretação dos dados, redação e revisão crítica,

\section{2 - Danielli Rafaeli Candido Pedro}

Contribuições: redação e revisão crítica.

\section{3 - Raquel Gvozd Costa}

Contribuições: obtenção ou análise e interpretação dos dados.

\section{4 - Paloma de Souza Cavalcante Pissinati}

Contribuições: obtenção ou análise e interpretação dos dados.

\section{5 - Mariana Ângela Rossaneis}

Contribuições: obtenção ou análise e interpretação dos dados.

\section{6 - Maria do Carmo Fernandez Lourenço Haddad}

Contribuições: concepção e planejamento do projeto de pesquisa, redação e revisão crítica.

\section{Como citar este artigo}

Cardoso MG, Pedro DRC, Costa RG, Pissinati PDC, Rossaneis MA, Haddad MCFL. Seguimento clínico laboratorial de trabalhadores que sofreram acidente com material biológico em instituições hospitalares. Rev. Enferm. UFSM. 2019 [Acesso em: Anos Mês Dia];vol9 e51: P1-16. DOI:https://doi.org/10.5902/2179769236110 